\title{
Peritoneal Fluid is Negative for Malignancy
}

National Cancer Institute

\section{Source}

National Cancer Institute. Peritoneal Fluid is Negative for Malignancy. NCI Thesaurus.

Code C160821.

A result that the peritoneal fluid is negative for malignancy. 